$\longrightarrow$

\title{
Targeting miRNAs in ALS
}

\section{By Tim Fulmer, Senior Writer}

A team from The University of Texas Southwestern Medical Center at Dallas has identified a muscle-specific microRNA-miR-206-that plays a role in the progression of amyotrophic lateral sclerosis. ${ }^{1}$ The results suggest that muscle cells could be therapeutic targets in addition to the more commonly pursued motor neurons. miRagen Therapeutics Inc. has licensed the findings and plans to design a therapeutic and eventually seek a partner to test it in the clinic.

ALS is an adult motor neuron disease characterized by atrophy and paralysis of lower limb and respiratory muscles. Nonfamilial, sporadic

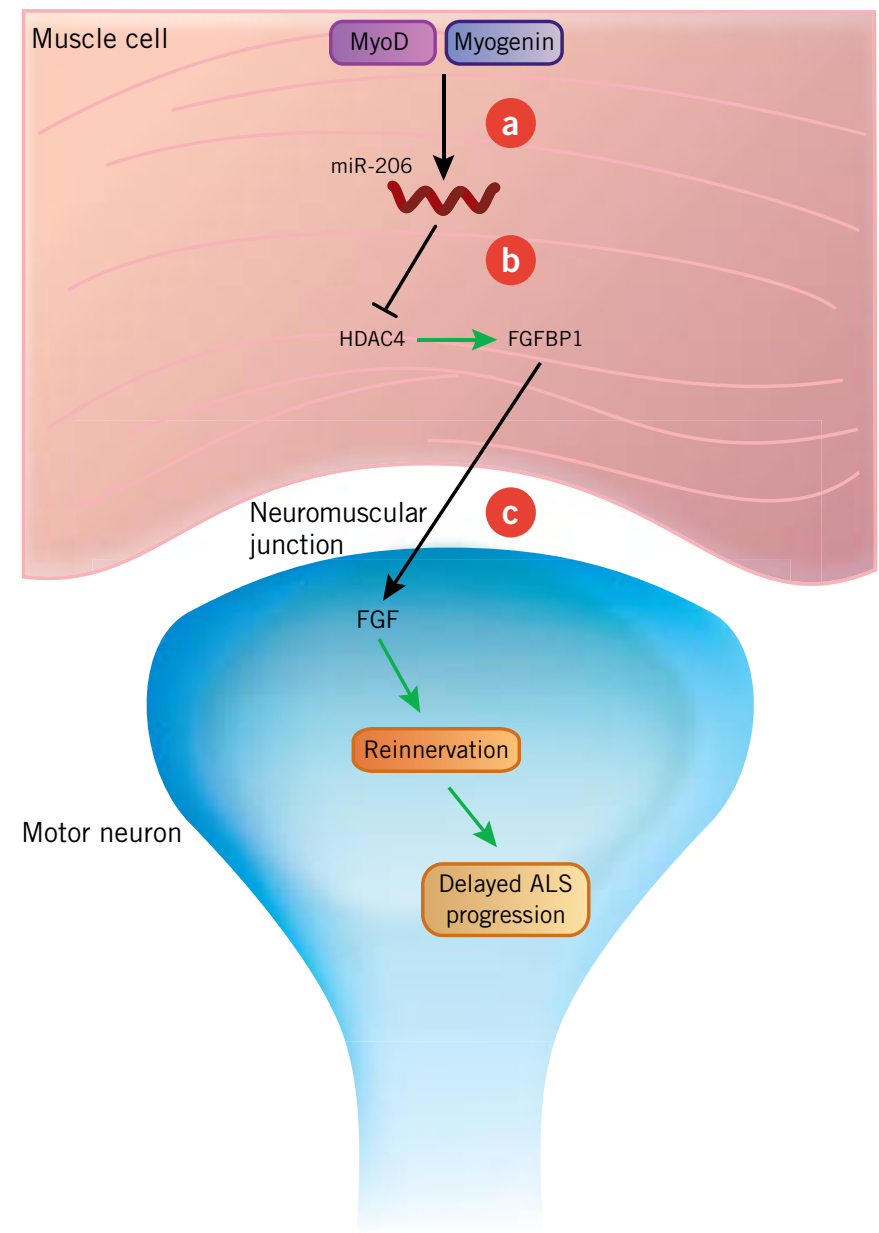

ALS is the most common form. Familial ALS occurs in about 20\% of patients, and about a quarter of those cases are linked to mutations in the gene coding for superoxide dismutase 1 (SOD1). Rilutek riluzole, a sodium channel blocker from sanofi-aventis Group, is the only approved treatment.

Previous work at UT Southwestern had implicated miRNAs in the stress response of cardiac muscle in myocardial infarction (MI) and other conditions. ${ }^{2}$ Eric Olson and colleagues hypothesized that miRNAs could be involved in the stress response of non-cardiac muscle that underlies conditions such as ALS.

His group studied miRNA expression in a transgenic mouse model, comparing levels of $320 \mathrm{miRNAs}$ in the lower-limb skeletal muscles of ALS mice and healthy controls. miR-206 was found to be the most significantly upregulated miRNA following onset of neurological symptoms $(p<0.0001)$.

The question was whether decreasing or further increasing miR-206 levels would slow or block ALS progression.

In the same models, knockout of miR-206 did not affect disease onset but did accelerate progression and reduce survival compared with expression of $m i R-206$.

Thus, the therapeutic goal would be to increase miR-206 levels to reduce disease progression.

Expression analysis of mouse muscle cells identified histone deacetylase 4 (HDAC4) as a downstream effector of miR-206. miR-206 at least partly mediated its effects by inhibiting expression of HDAC4, a protein known to counteract muscle reinnervation by blocking expression of

Figure 1. The role of miR-206 in ALS. Researchers at The University of Texas Southwestern Medical Center at Dallas have identified microRNA-206 (miR-206) as a key player in the regeneration of the neuromuscular junction in mouse models of amyotrophic lateral sclerosis (ALS). The latest findings, published in Science, suggest miR-206 mediates its effects via the following mechanism:

[a] In response to skeletal muscle denervation that can occur during injury or progression of neuromuscular diseases like ALS, myogenic regulatory factors (MRFs) such as MyoD and myogenin are upregulated in muscle cells.

[b] The increased levels of MRFs activate transcription of miR206, which then binds mRNA encoding multiple proteins, including histone deacetylase 4 (HDAC4), and blocks expression of those proteins. One consequence of blocking HDAC4 expression is greater downstream synthesis of fibroblast growth factor binding protein 1 (FGFBP1).

[c] FGFBP1 then binds and activates FGF proteins, which promote reinnervation and regeneration of the neuromuscular junction, thus delaying ALS progression and prolonging survival.

The UT Southwestern team is collaborating with miRagen Therapeutics Inc. to create miR-206 mimetic compounds that could help regenerate neuromuscular junctions damaged in ALS. 
fibroblast growth factor binding protein 1 (FGFBP1; see Figure 1, “The role of miR-206 in ALS”).

The researchers also looked at whether miR-206 was upregulated following denervation of skeletal muscle, a process that underlies ALS. Indeed, 10 days after surgical denervation of the lower leg muscles of wild-type mice, miR-206 levels were higher than those in control animals that had not undergone denervation.

In healthy mice subjected to surgical denervation, animals with miR-206 knockout had delayed reinnervation of muscles compared with wild-type mice, suggesting that miR-206 could play a protective role in ALS.

Taken together, these experiments suggest that miR-206 helps trigger regeneration of the junction between motor neurons and muscle cells after nerve injury and that enhanced regeneration of the neuromuscular junction was at least partly responsible for slowing disease progression.

The results were published in Science, and Olson was the corresponding author on the paper.

"The elegant study by Olson and colleagues shows that muscle cells are potentially as important a target as motor neurons for developing ALS treatments. Indeed, the paper identifies multiple therapeutic targets in muscle, including miR-206 itself and its downstream effectors such as HDAC4 and FGFBP1," said Philip Gregory, CSO and VP of research at Sangamo BioSciences Inc.

"It would now be useful to show that enhancing miR-206 levels in disease-either by activating expression of endogenous miR-206 or by adding back an miR-206 mimetic-results in a robust, long-term treatment effect in ALS animal models," Gregory said.

Sangamo's SB-509, a plasmid DNA encoding a zinc finger DNAbinding protein transcription factor (ZFP TF) to upregulate VEGF-A gene expression, is in Phase II testing to treat ALS.

\section{Designing a therapeutic}

Olson is also a cofounder of miRagen, which has exclusively licensed IP covering the findings in the Science paper. He told SciBX the company is seeking "to develop miR-206 mimics to enhance motor neuron regeneration."

miRagen cofounder, president and CEO William Marshall said the company plans to "design and synthesize miRNA therapeutics that mimic the function of miR-206 in ALS. We'll subsequently look for academic and/or company partners with ALS expertise to help us test candidate therapeutics in additional ALS preclinical models and eventually move the most promising candidate into the clinic."

Olson said he wants to look for additional therapeutic targets involved in miR-206 signaling. "We are working on strategies to manipulate signaling pathways downstream of miR-206 in the setting of ALS and other neuromuscular diseases," he said.

miRagen has multiple miRNA-targeting compounds in preclinical development to treat cardiovascular indications including chronic heart failure (CHF), post-MI remodeling and cardiac fibrosis.

Fulmer, T. SciBX 3(1); doi:10.1038/scibx.2010.2

Published online Jan. 7, 2010

\section{REFERENCES}

1. Williams, A. et al. Science; published online Dec. 11, 2009; doi:10.1126/science.1181046

Contact: Eric Olson, The University of Texas Southwestern Medical Center at Dallas, Dallas, Texas e-mail: eric.olson@utsouthwestern.edu

2. van Rooij, E. et al. J. Clin. Invest. 117, 2369-2376 (2007)

\section{COMPANIES AND INSTITUTIONS MENTIONED}

miRagen Therapeutics Inc., Boulder, Colo.

Sangamo BioSciences Inc. (NASDAQ:SGMO), Richmond, Calif. sanofi-aventis Group (Euronext:SAN; NYSE:SNY), Paris, France The University of Texas Southwestern Medical Center at Dallas, Dallas, Texas 